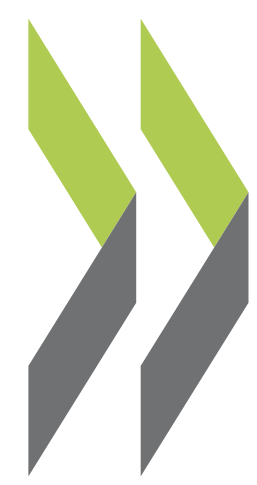

PEB Exchange, Programme on Educational Building 2003/03

Review of Building Quality Using Post Occupancy

\author{
Chris Watson
}

\title{
Evaluation
}

https://dx.doi.org/10.1787/715204518780 


\section{REVIEW OF BUILDING QUALITY USING POST OCCUPANCY EVALUATION}

\section{Introduction}

"Darkrooms should be dark" was the observation of a teacher reviewing school photographic facilities in a Post Occupancy Evaluation conducted by Chris Watson. Occupants' indisputable, but unfulfilled, expectations of building quality are documented within the recorded opinion in Post Occupancy Evaluations. In this case, glazing in outer doors compromised the light seal of the darkroom lobby. Such gross errors in building production are relatively rare, but they serve a useful reminder of the importance of systematic review to reduce the likelihood of repetition and ensure their correction.

Post Occupancy Evaluation is described here in terms of its aims, principles, benefits, methods, timing and results. Three examples illustrating the evaluation of educational facilities are given, followed by a summary.

\section{Aims}

Education depends on students being supported, not frustrated, by buildings and equipment. Classroom communication requires certain acoustic, visual and physical conditions, and feedback on efficient design is essential for improving future design. By understanding how existing buildings affect occupants, designers can minimise problems and capitalise on successful design features.

Post Occupancy Evaluation is a tool to account for building quality - essential when organisations are required to demonstrate that building programmes are being responsibly managed.

Participants in Post Occupancy Evaluation often identify ways to design and use buildings and equipment more efficiently and more cost-effectively. Dysfunctional or seldom-used building features can be identified and eliminated from future designs. Imperfections in new buildings can be fine-tuned and management practices adjusted. Often, minor changes to buildings and the ways they are used offer significant benefits to users.
Systematic analysis of buildings from all relevant viewpoints is also useful for negotiating between the various interest groups and assists them in realising the potential and limitations of their buildings. Post Occupancy Evaluation involves the building users in defining how buildings function for them. This participation has been shown to engender greater commitment to solutions and more willingness to accept shortcomings.

Evaluation is also an important tool in planning refurbishment of existing buildings. It helps clarify perceived strengths and weaknesses in order to focus resources where they are needed. It identifies where building design adjustments are needed to support changing practices, markets, legislation and social trends.

\section{Principles}

On the basis that buildings are socially negotiated solutions to human needs for shelter (and support for operations and equipment), no objective method of evaluation can be a complete test of building quality. Consequently, the evaluation compiles the subjective opinions from the complete set of interest groups.

Once all the relevant occupant groups have been identified, they collectively are the "experts" at knowing how the building affects their activities, needs and aspirations. The approach taken is then to capture as much opinion as possible about the subject building.

To ensure that buildings are interpreted in the manner appropriate to each occupant group and that the evaluation remains free of evaluator bias, participants are invited to respond directly to spatial experience in the building, rather than any evaluator representation of it.

Post Occupancy Evaluation reports are compiled to ensure easy review for the various parties who need to refer to different types of recommendations within it. The reports need to be transparent in their logic so all parties can understand and engage in healthy debate and negotiate on people-centred environments.

Lastly, evaluations are helpful when they are enjoyable and empowering to participate in, and when they deliver prompt results.

\section{Benefits}

Organisations that commission Post Occupancy Evaluations are generally experienced and competent to achieve satisfactory buildings, so evaluations are usually focused on fine gains of relatively few design issues. 
These organisations employ Post Occupancy Evaluation to:

- account for building quality;

- save money;

- identify successful design features to repeat;

- identify problems to mitigate or reduce;

- fine tune completed buildings;

- identify redundant or unnecessary building features;

- empower users to negotiate building issues.

\section{Methods}

The first, and perhaps most crucial step, in evaluating buildings is to identify the people they affect. This group may include students, teachers, school managers, government education specialists, the project team and those who maintain and clean the buildings.

Groups of five to seven individuals with like interests are firstly given a ten-minute introductory meeting in preparation for a touring walkthrough interview. The walkthrough explores all parts of the facility the participants wish to comment on and typically takes 30-90 minutes. Priorities are established in a review meeting.

Participants' comments are documented and building features are photographed. Recommendations are then developed that are applicable both to future buildings and for fine-tuning the subject building.

Additional methods include structured discussion forums, observation studies (timed position plotting and tracking studies), questionnaires and expert scans.

\section{Timing}

A typical evaluation requires approximately two months, counting a couple of weeks to invite participants, up to one week for walkthrough interviews on site, followed by three to six weeks to prepare the report.

Post Occupancy Evaluation can occur at any time in the life of a building. An evaluation of one major building was undertaken two months before formal opening. Routine Post Occupancy Evaluation programmes review buildings six to 12 months after completion. Buildings are often reviewed as part of the programming/briefing for alterations.

\section{Results}

Each Post Occupancy Evaluation includes written and photographic documentation of participants' opinions about how the facilities affect them. These opinions, col- lated in groups of emerging issues, are then presented along with the generated recommendations to allow transparent "logic" of evidence for each issue.

Recommendations can be one of four types, namely:

- "now" for actions recommended within three months;

- "later" for actions recommended within three years;

- "future" for actions or practices recommended on future buildings;

- "focus study" for technical studies or management decisions.

\section{Example 1: School technology centre}

Built in 2000, the Marlborough Technology Centre was the first school workshop building designed to support New Zealand's national technology curriculum. It is used by more than 1000 school children from throughout the surrounding province for food, video, biology, textile and hard material technology. A Post Occupancy Evaluation was commissioned by the Ministry of Education to identify strengths and weaknesses before a similar design was replicated at schools in other regions.

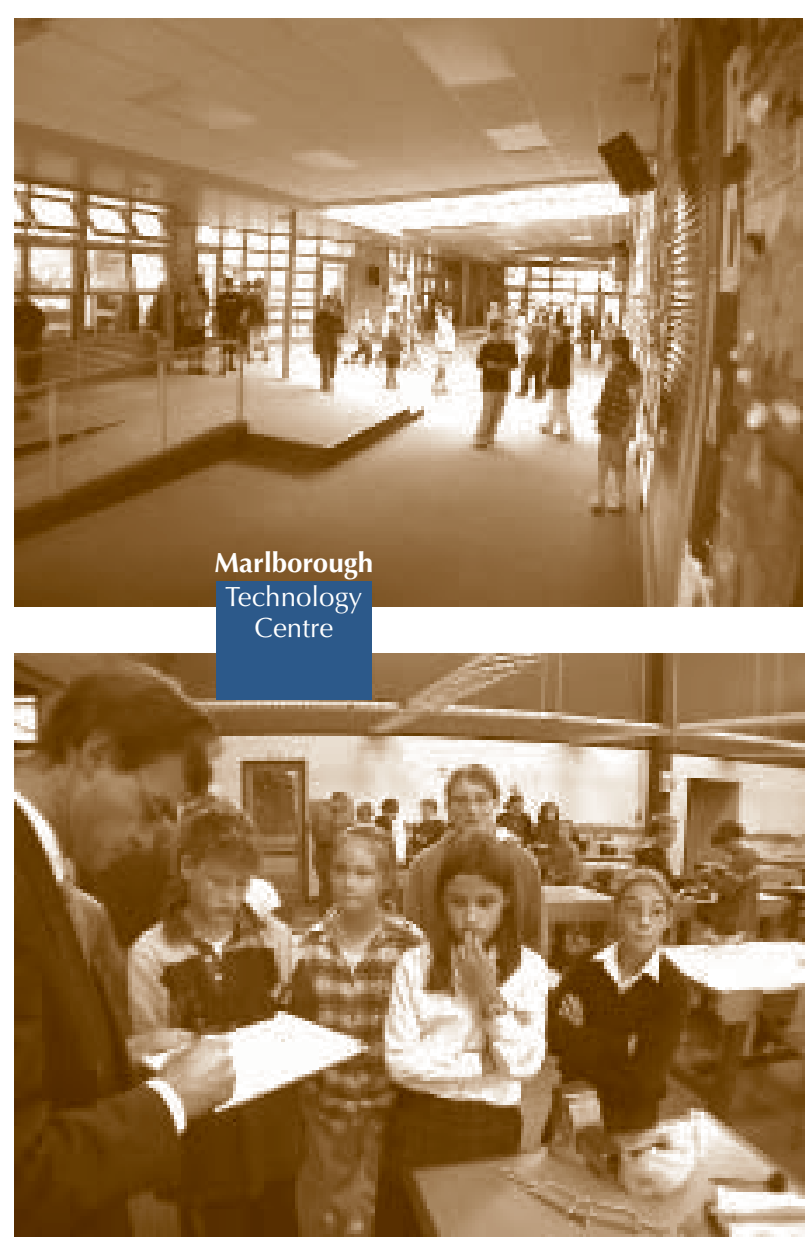




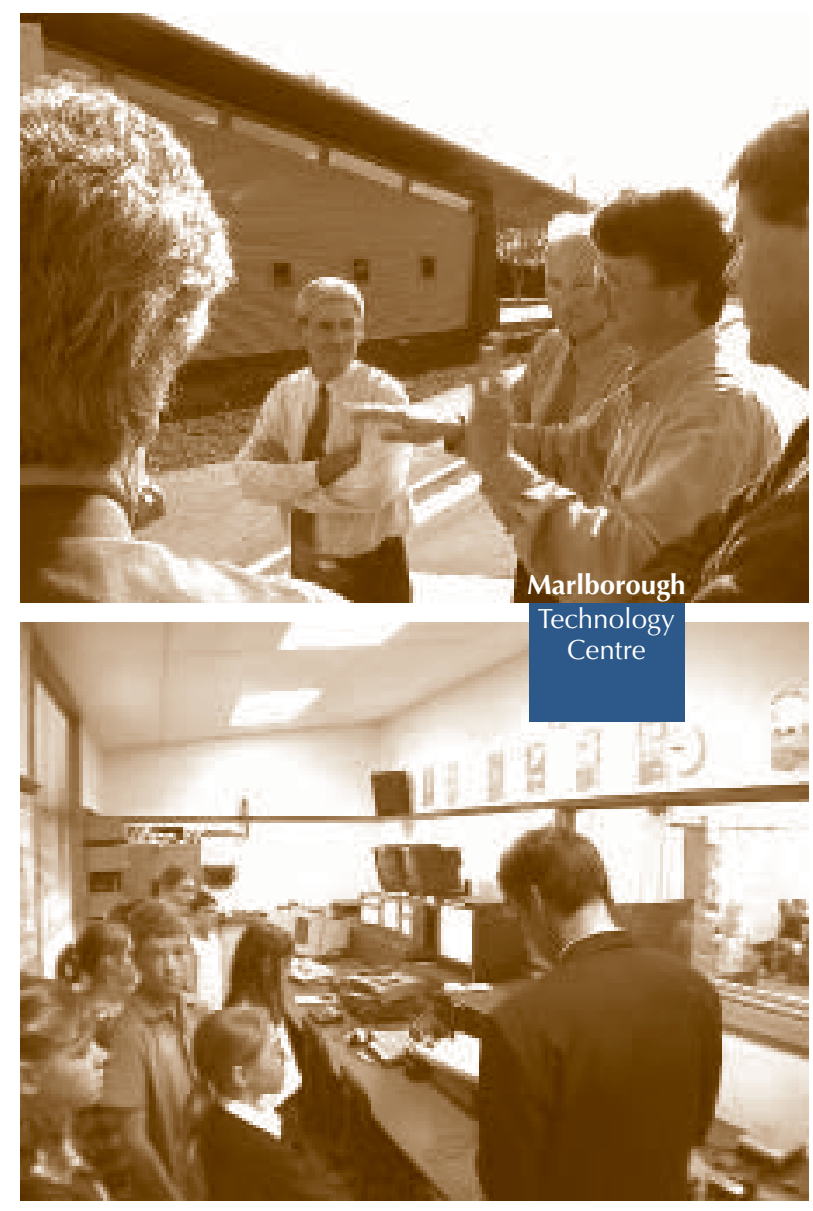

Groups of students, teachers, the project team and education specialists were interviewed using the touring walkthrough method. On the second of the two-day Post Occupancy Evaluation, selected participants developed their ideas further in a planning forum.

The evaluation found that the centre successfully supports student learning, and it produced three key recommendations. The first of these is related to problems associated with the building's multiple uses, including noise control. The consultant and participants identified relatively simple design modifications to solve these issues. Secondly, the new technology curriculum and building model required changes in teaching culture and interschool co-operation. Another important recommendation was the need for input from a wide range of stake-holders when similar buildings are being planned.

For the Ministry, Post Occupancy Evaluation represented a systematic way to identify the effects of the new facility on student learning. "Post Occupancy Evaluation definitely helped us identify a measurable link between building quality and educational outcomes, which is notoriously difficult to show," said John MacDuff, District Manager of the Ministry of Education. "The evaluation was a very helpful way for the local management team and me to bed-in systems, and crucial in preparation for designing the next technology centre."

\section{Example 2: Student accommodation building}

The Railway Campus opened in 1999 in the extensively restored former central railway station and can house up to 630 students in 230 residential apartments. Uni-Accommodation Limited, a wholly owned trading subsidiary of The University of Auckland operates the accommodation and food and beverage activities of the strata titled development. In 2001 the university undertook a Post Occupancy Evaluation to improve the ongoing operation of the complex and to help plan future accommodation initiatives.

Building users were generally pleased but saw scope for improvement in areas including security, computer facilities, bird-proofing, ongoing restoration and repair, and tenancy agreements.

Design and management strategies were recommended to address problem areas. The Post Occupancy Evaluation also identified several important issues for consideration when planning future student accommodation, including the need for level access and good acoustic conditions. The evaluation was subsequently used as the basis for a review of another 800-bed student accommodation proposal.

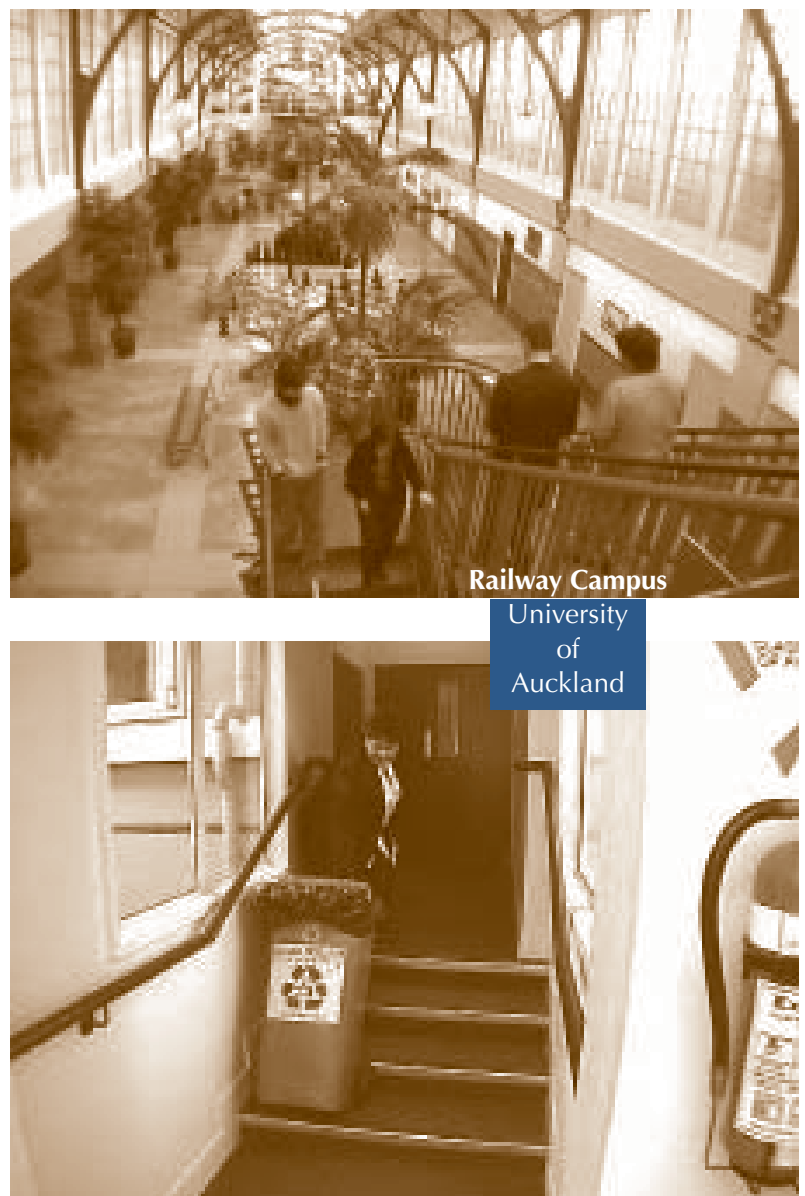




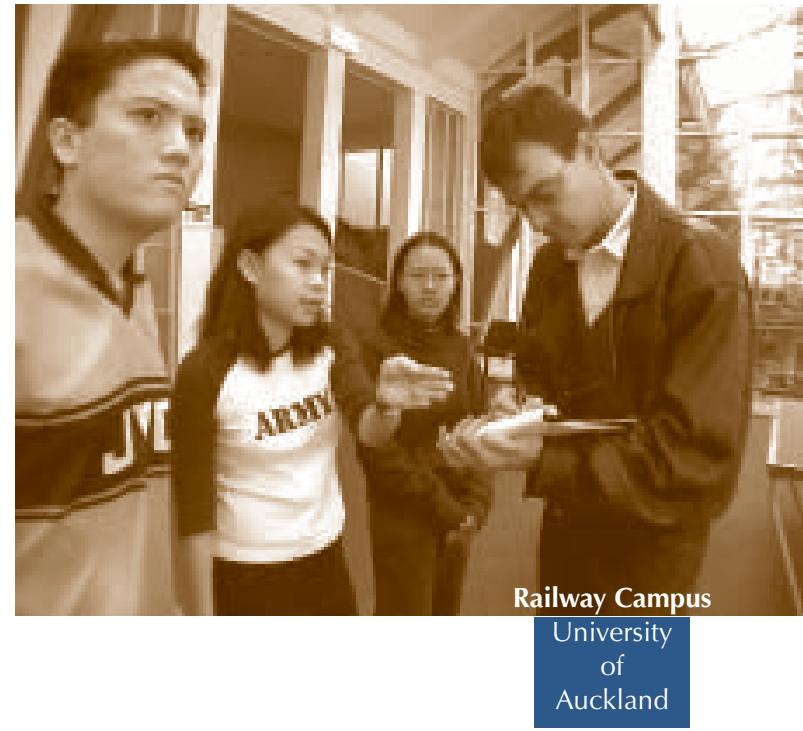

Stewart McElwain, Group Manager of Student Affairs at the university, said Post Occupancy Evaluation delivered three key benefits: "Firstly, it was a fantastic opportunity for student residents to communicate their experiences and perceptions first-hand, and for them to see we were prepared to make design and management improvements. The Post Occupancy Evaluation also helped us in our ongoing relationship with the building investors who own the apartments. And finally, we were able to apply our better understanding of the building's strengths and weaknesses to decisions we were making about a new student accommodation facility."

\section{Example 3: Multiple school building Post Occupancy Evaluations}

During 2000-2001 a set of 20 Post Occupancy Evaluations were conducted on typical small to medium building projects managed by schools, including site services, refurbishment of a few rooms, a new administration building, renovation of small schools and new libraries. Each school self-managed its own project with local consultants and received government funding.

Evaluation of these projects was commissioned by the Ministry of Education to assist schools to learn from others that have previously undertaken similar work. Schools working on building projects can now download the evaluations of relevant projects from the Internet.

The matters raised in the evaluations include both project issues and design issues that became apparent after using the project for a few months. An important feature for the Ministry of Education was the satisfaction rating participants applied to the project and the completed building.
One of the particularly successful projects was the Kaikorai Primary School administration/library facilities that were found to have been well tailored to integrate with existing buildings. The highly-resolved design solution provided flexible and cohesive support for learning.

In a further development of this evaluation programme, the first school has piloted a do-it-yourself Post Occupancy Evaluation. This first evaluation, of a science block, had mixed results. The school experienced some difficulties with participation and presentation but was able to establish fine tuning priorities to its new building. Further monitoring of this process will be ongoing.

\section{Summary}

As design is a socio-political process rather than an absolute science, Post Occupancy Evaluations are managed as communication forum in support of design to negotiate the relationship between people and buildings. With changing and increasingly complex building requirements, good communication is essential to align facilities with learning requirements.

Building users, designers and owners profit from Post Occupancy Evaluations. Building users say they benefit directly from being empowered to comment on their built environment. If designers have building users' opinions in an appropriate format then they can reduce the "second guessing" about what is important to occupants. Building owners report that occupant participation helps them accept facilities and adjust their practices to suit new buildings.

Post Occupancy Evaluation provides a systematic way of learning from successes and mistakes of previous buildings. It then offers that information in a timely and appropriate way to improve future buildings and to account for design quality of educational buildings.

Chris Watson is an architect with specialist experience in approximately 100 Post Occupancy Evaluations since the early 1980s. He has conducted reviews of schools, universities, apartments, courts, banks and museums, together with facilities for the military, petrol retailing, prisons and archives. In 2000, he advised PEB delegates at the Paris conference on Post Occupancy Evaluation. Further information about his international practice is available at http://www.PostOccupancyEvaluation.com or by e-mailing. chris@PostOccupancyEvaluation.com. 